\title{
Pinocytotic Vesicle
}

National Cancer Institute

\section{Source}

National Cancer Institute. Pinocytotic Vesicle. NCI Thesaurus. Code C33324.

A vesicle formed by the fusion of the plasma membrane, which captures extracellular fluids and solutes during pinocytosis. 